\title{
The Design of Embedded Remote Intelligent Automotive Monitoring System based on GSM
}

\author{
Lijun Gao \\ Beijing Information technology college, Beijing 100018, China \\ gao@bitc.edu.cn
}

\begin{abstract}
Keywords: Embedded, remote intelligent, automotive monitoring system, GSM, automotive
\end{abstract} microcontroller.

\begin{abstract}
The paper proposes a remote intelligent automotive monitoring system based on GSM network and designs the hardware and software work after designing the system process. PC automotive monitoring center, MCU, peripheral interface circuits and GSM communication module which used to relate PC and MCU are built for this system which uses GSM network as a wireless remote data transmission platform, and then highlighted the relative importance of this subject of GSM network short message communication principles.
\end{abstract}

\section{Introduction}

With the development of modern communication technology, GSM mobile communication has a very wide range of applications, [1] the GSM short message system which based on the TDMA technology is more mature, improve, and get an extensive application. GSM short messages can be made into various functions such as transport a wide variety of testing, automotive monitoring, data communication signaling and control command system, [2] widely used for remote automotive monitoring, GPS navigation, and other personal communications terminal areas.

In recent years, with the development of network technology, control technology and life standard and quality, information technology is changing people's work habits and lifestyle. So in recent years, remote control system, [3] intelligent Home Furnishing system has been widely used in people's life and work, at the same time also put forward a very high request to performance, power consumption and stability of the remote control system's general remote control system consists of the internal network, the external network, the gateway and the terminal application program, and among them the internal network and the gateway is the core part of remote control system.

\section{Hardware}

The appearance of the wireless monitoring system combined with GSM/GPRS networks supply the gap of the systems based on other communication networks. Therefore, the article researches and produces the monitoring system which is through the Short Message Service based on GSM networks and combined with the wireless data-transfers of GPRS networks. [4] This system solves the above problems and with characteristics of low-cost, upgradeable and easy-extended. It can meet the need of the private users as well as the small-scale users, so, there must be a brighter future for this system.

In the design of the hardware, MSP430F149 with huge internal resource and ultra-low power characteristics is used as the CPU, MCU and GSM wireless communication module produced by Germany's Siemens company, are used to send and receive short messages. [5] The whole hardware structure can be divided into the host computers part and the MCU peripheral interface part. The host computer is a simple part, includes a PC and GSM module TC35, both through the RS232 serial port connection, MCU and peripheral interface part include TC35 communications module, LCD liquid crystal display unit, a variety of sensors data acquisition and processing units. 


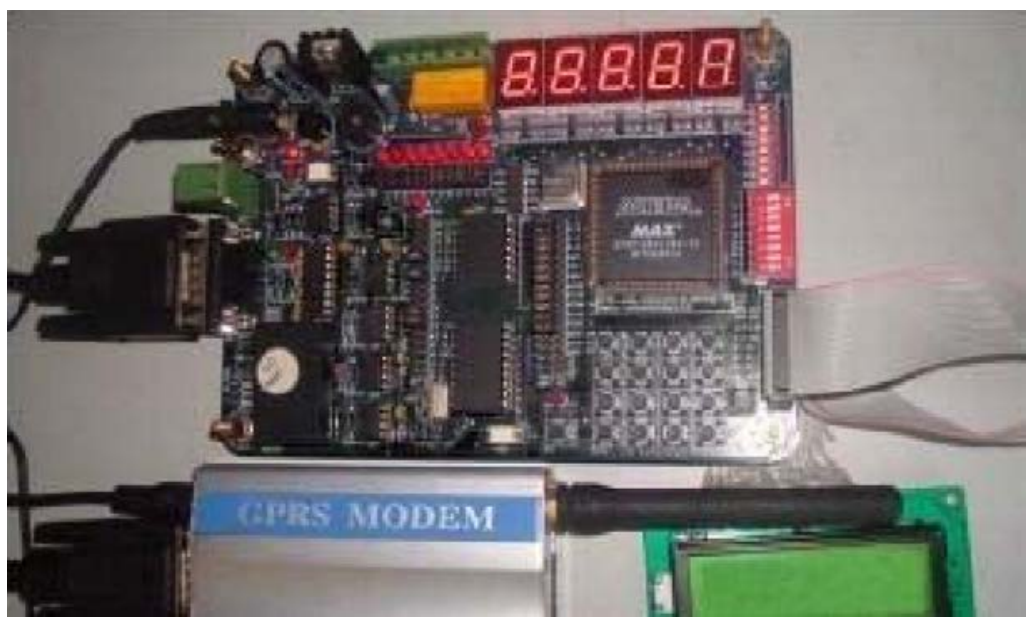

Fig. 1 Physical hardware circuit diagram

This paper designs a simple and practical remote intelligent control system. [6] The design takes the Cortex-M3 as the core processor, embeds UC/OS II as the instant operating system, uses CGI technology to complete the construction of the web server, transplants TCP/IP protocol stack, executes access and control via landing server in Internet, e. g. SMS protocol structure is shown in Fig. 2. The article uses the wireless module to form a wireless control terminal network, and control terminal collects the data and information to implement intelligent control through the DS 18B20 sensors. Users can login server through the web browses of the mobile terminal for automotives out monitoring and control to the remote control terminal.

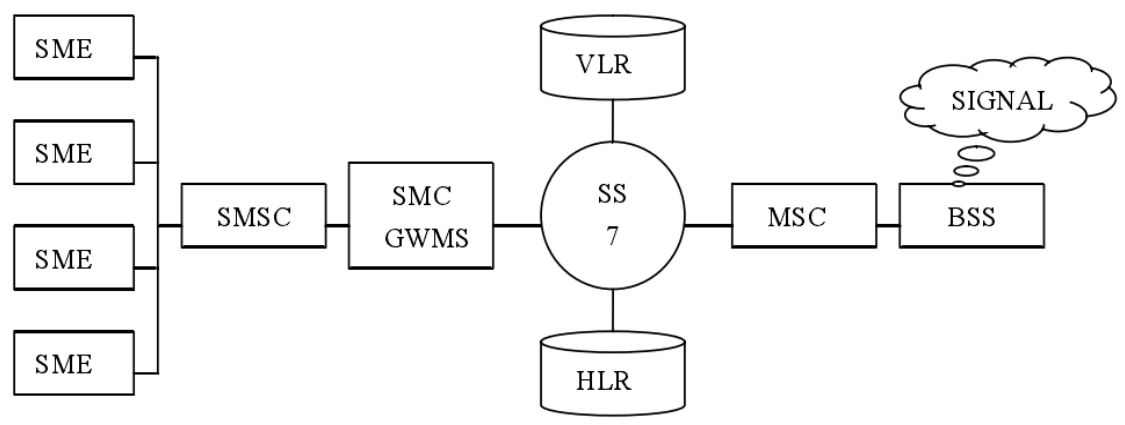

Fig. 2 SMS protocol structure

\section{Software}

In system software design, PC software using the Visual Basic 6, adding MSComm ActiveX, to complet the computer interface, and deal the standard AT command. In the design of MCU and peripheral interface, using IAR EW430 integrated development environments and c language debugger as the MSP430 microcontroller program development and debugging tools, its software program includes system initialization, interrupt signal acquisition and A/D conversion process, short message sending and receiving and so on. 


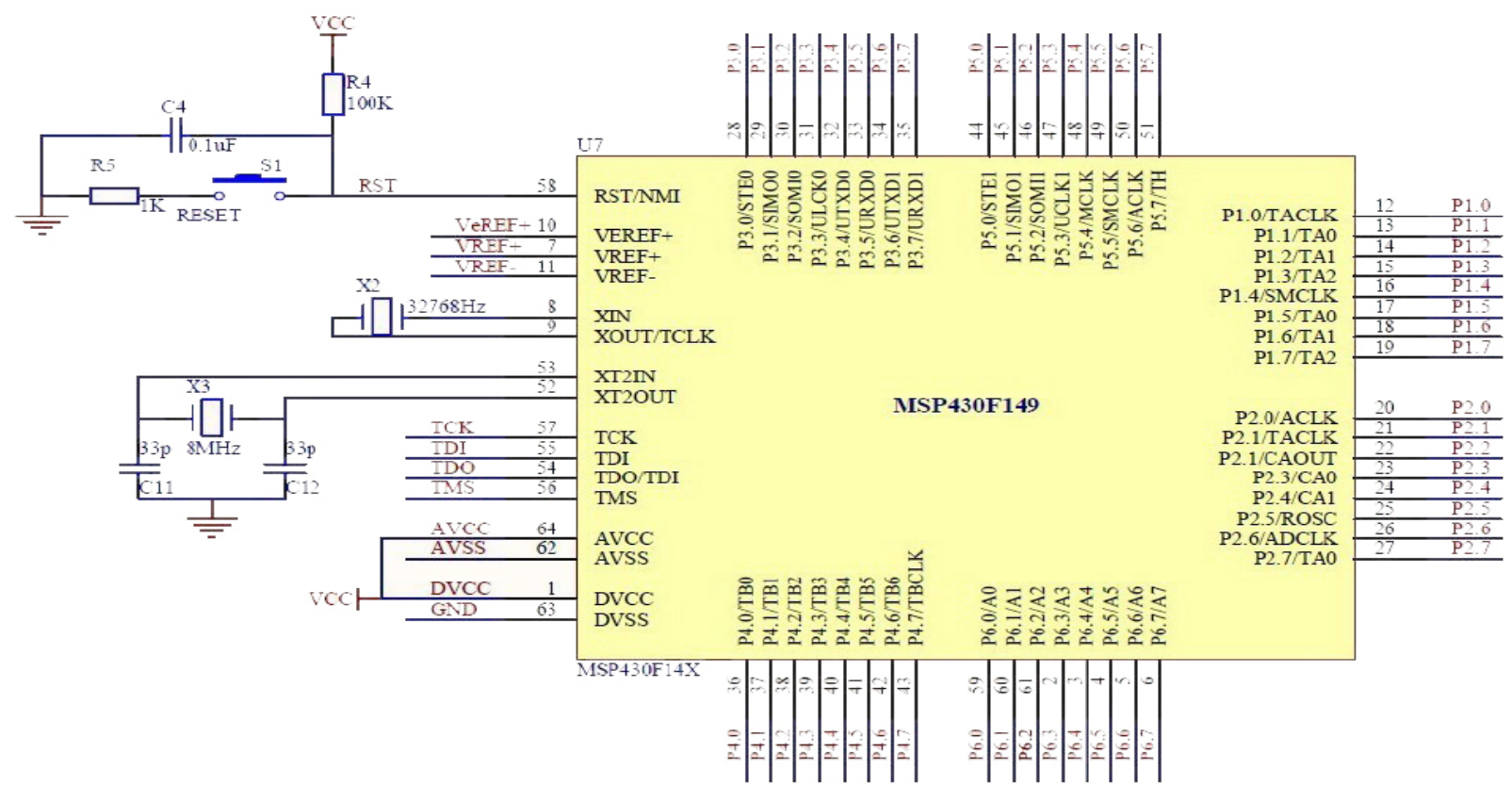

Fig. 3 Single chip microcomputer minimum system design

By using S3C6410 hardware platform and Linux OS, as well as GPRS module to build terminal monitoring platform which is to receive and processes and analysis of data collected from various sensors, meanwhile send the information parsed commands from smart phones monitoring client to various sensors collect points.

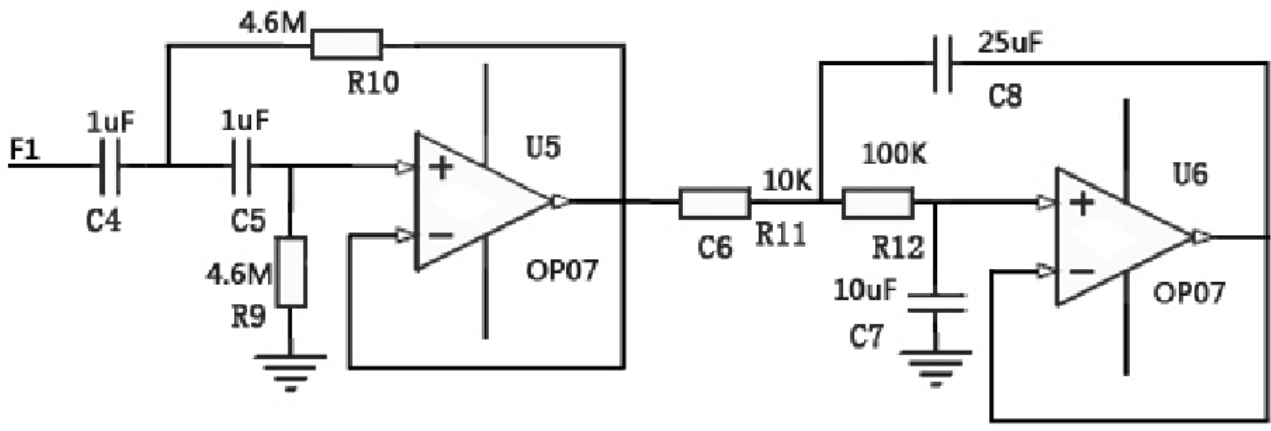

Fig. 4 Filter circuit design

Filter circuit design is shown in Fig. 4. Where, the output voltage is:

$R 2=R 1\left(\frac{\text { VOUT }}{1.216}-1\right)$

$R 1$ and $R 2$ express resistance value. So the cutoff frequency expresses:

$$
\begin{aligned}
& F 2=\frac{1}{2} \pi \sqrt{C 6 C 7 R 11 R 12} \approx 100.7 \mathrm{~Hz} \\
& F 1=\frac{1}{2} \pi \sqrt{C 4 C 5 R 9 R 10} \approx 0.03 \mathrm{~Hz}
\end{aligned}
$$

\section{Monitering system design}

The research and implement of Auto remote intelligent control system takes good use of multidisciplinary and technological achievements of the Global Satellite Positioning System, Geographical Information System and Single-chip Microcomputer Control field. Based on intelligent mobile terminal control, this system is mainly used for teaching and scientific researching to meet the lower market requirement. It can be widely used in car real-time monitoring, driving region restricting, lock and unlock vehicle. Besides, extended channel is provided for customization. This system is good for teaching and scientific researching and has high value of economic benefits. 
The system using GSM short message networks as a wireless data connection base, combined with the ECG abnormal alarm monitoring, designed of the system based on GSM short message network to realize the remote ECG monitoring. After experimentally tested, the system runs stable, live strong, and achieve the desired result. Compared with conventional ECG machine, not only by low power consumption, small size, and simple operation and easy to carry, but also greatly reduces the cost of ECG monitoring system. The system has some practical value.

Firstly, the thesis introduces the overall design of the ARICS(automotive remote intelligent controller System) which including the full range of market demands, teaching requirements and its design principles, and thus presents a complete design solution. Secondly, we design the vehicle-side intelligent controller hardware selection and key module design, detailed description of the systems theory, control module design, GPS positioning module design, GPRS communication module hardware selection and circuit design. Then we analyze the handheld software control terminal for intelligent controller of car remote control and com out the design, analysis of the software systems development and integration environment, then gives the software design of the overall system framework and functional modules, detailing introduce the database, the zone control function, the lock and unlock control module, extended channel control module, positioning module, tracking module functional, management module and the user interface module.

Finally, we did testing and real testing and conduct a systematic analysis, summarized and pointed out the shortcomings of the system, prompt the improved method.

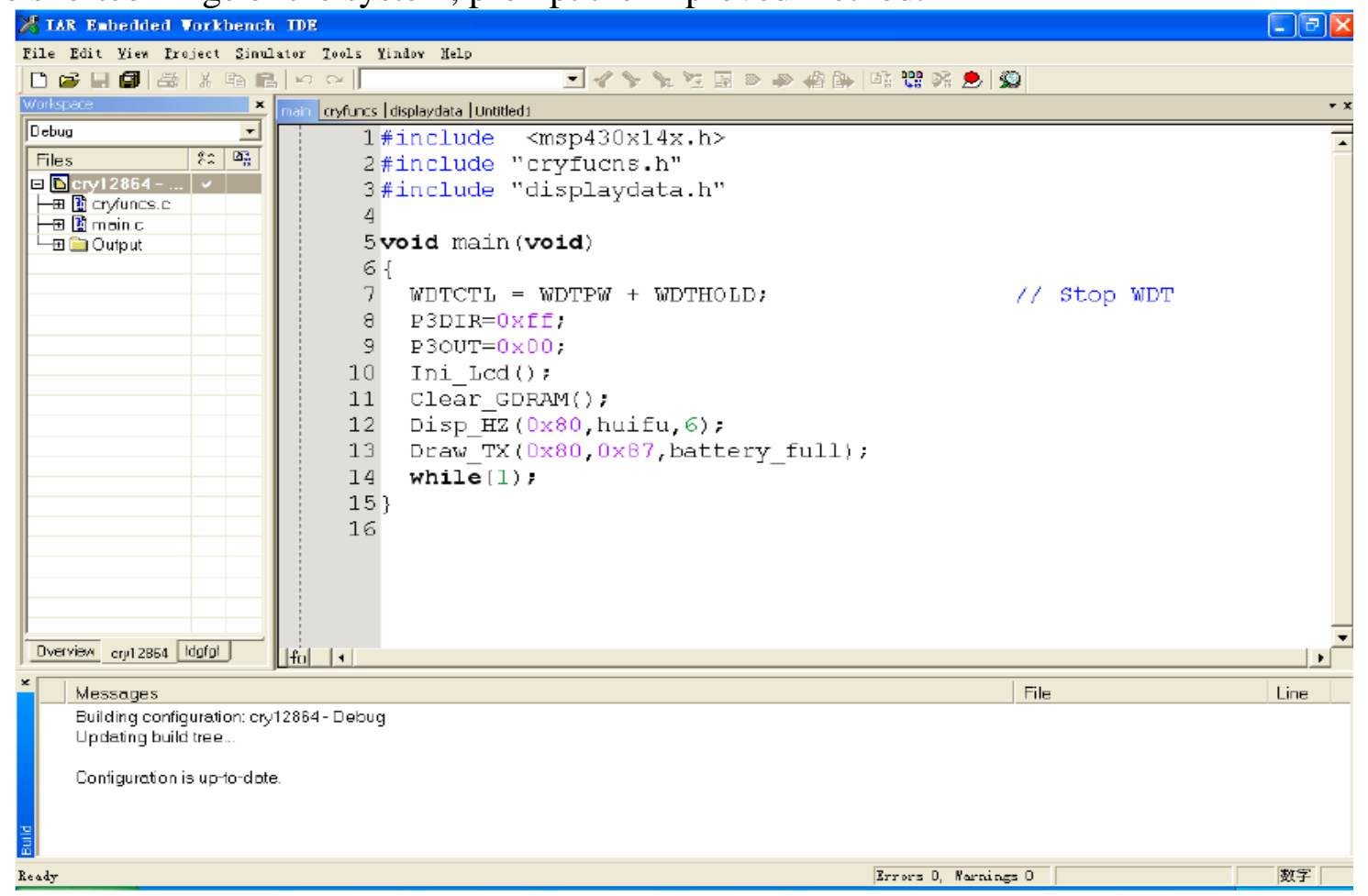

Fig. 5 MCU development of debugging and test interface

MCU development of debugging and test interface is shown in Fig. 5. The system has stable performance, good real-time performance and low design cost, is advantageous for the application in intelligent Home Furnishing, remote monitoring and control in industrial and many other fields, also has wide application prospect.

\section{Conclusions}

A design and application of embedded system are introduced in long distance intellectual monitoring of automobiles that embedded-system is used into the guard against theft of automobiles for the first time. The intellectual guard against theft, remote control area location and dispatch of mobile phone, telephone, Internet and automobiles are achieved by GSM/GPRS and GPS. The system has certain value in practice. 


\section{References}

[1] Li C,Zheng C, Tai C. Detection of ECG characteristic points using wavelet transforms. IEEE Transaction on biomedical, 2008, 42(1): 21-28.

[2] M Tang, C Chou, C Hui Tang ,et a1. Exploiting GSM short Message Service for Ubiquitous Accessing.Journal of Network and Computer Applications, 2001, 24: 249-267.

[3] S. Goel and D. Duchamp. Linux Device Driver Emulation in Mach. Proceedings of the Annual USENIX 2012 Technical Conference, San Diego, CA, USA, Jan. 2012: 65-73.

[4] A.P.M. Michel, K. L. Cro, K. W. McLetchie. A Remote Monitoring System for Open Ocean Aquaculture. J.D. Irish Woods Hole Oceanographic Institution, 2014, 12(1): 69-72.

[5] Gunawardna Sanjeev ,Remote-controlled continuously operating GPS anomalous event monitor. 19th International Technical Meeting of the Satellite Division, 2006, 5: 381-392.

[6] Jiang min Qin. Design and research on the remote monitor system based on embedded Internet. 2006 1st IEEE Conference on Industrial Electronics and Applications, 2006, 1: 402-406. 